\title{
Parametric Analysis of Piezoelectric Resonances of AIN Layouts for Vibrating Energy Harvesting
}

\author{
Alessandro Massaro ${ }^{1, *}$ and Roberto Cingolani ${ }^{2}$ \\ ${ }^{1}$ Italian Institute of Technology IIT, Center of Bio-Molecular Nanotechnology, Arnesano, 73100, Italy \\ ${ }^{2}$ Italian Institute of Technology IIT, Via Morego 30, Genova 16163, Italy
}

\begin{abstract}
In this paper, we perform an accurate parametric analysis of piezoelectric cantilevers for micro-scale vibrating energy harvesting applications. The presented model considers the electromechanical coupled equations including the losses due to the damping effect and losses of internal frictions. By assuming an in-phase sinusoidal source as external applied vibration force, the model studies the best electromechanical efficiency by analysing the optimal load resistance for a piezoelectric aluminum nitride (AIN) cantilever operating around $1100 \mathrm{~Hz}$. The approach of the model allows to define the design criteria for efficient vibrating energy harvesting AIN cantilevers according with the actual technology. The model is extended to ring layouts able to work at lower frequencies.
\end{abstract}

Keywords: Cantilever, Energy Harvesting, Analytical Modeling, Integrated Harvesting System, Damping Losses, MEMS.

\section{INTRODUCTION}

Over the past few years, the development of energy harvesting devices from various ambient energy sources has been the subject of intense investigation. Among the others, mechanical vibrations are easily exploitable through micro-electro-mechanical-systems (MEMS) technology. ${ }^{1-5}$ Mechanical vibration energy can be converted into electric energy by piezoelectric transducers, which are preferable to electromagnetic and electrostatic transducers due to their higher energy density. ${ }^{6}$ Piezoelectric mechanical transducers are based on the mechanical deformations undergone by bimorphic structures (piezoelectric/structural layer) induced by the internal stress generated in the piezoelectric material. The internal stress in the vibrating structure may have influence on the dynamic behavior, and hence on the deflection amplitude and on the generated power. In fact, vibrating energy transfer giving maximum power output occurs under resonance condition, for which the deflection amplitude is maximized. So far, several vibration harvesting devices have been reported ${ }^{2,3,7,8}$ which were mainly based on different piezoelectric structure designs and different interface circuits. However, for low vibration frequencies and high vibration amplitudes the use a proof masses has become necessary. ${ }^{9-13}$ Different vibrating energy harvesting models regarding the electromechanical coupling are presented in literature,${ }^{14-16}$ and,

*Author to whom correspondence should be addressed. usually the analysis considers approximations concerning the losses of the vibrating system. These approximations do not describe the real efficiency of the device and could provide wrong results. For this purpose we implement in our electromechanical model the most important contributions of losses for a cantilever vibrating system such as beam/air damping, proof mass/air damping, and finally internal frictions. ${ }^{17}$ According with a simple technology ${ }^{18,19}$ the accurate model presented in this work will be applied to an Alluminium nitride (AlN) piezoelectric cantilever. In the last years, piezoelectric AlN has become important thanks to its compatibility with conventional silicon technologies; it can be grown through a low temperature process and does not introduce defects, avoiding thus the major problems arising with other piezoelectric materials. Piezoelectric properties of AlN are similar to those of Zinc oxide ( $\mathrm{ZnO}$ ), but not as good as those of Lead Zirconate Titanate (PZT), however, its high elastic constants and elevated thermal and chemical stabilities make this material suitable for sensor applications including mechanical energy detection from vibrations or movements. The vibrations can be transformed into useful electrical power by using various electro-mechanical transduction schemes. Piezoelectric vibration energy harvesters typically consist of a bulk mass attached to a free standing structure such as cantilevers. These devices are based on a resonance principle (natural mode resonance) and they give maximum power output under their resonance conditions, for which the deflection amplitude is 
maximized. Traditional numerical methods such as Finite Element Method (FEM) or Finite Difference Time Domain (FDTD) can provide convergent solutions related to output powers, but they do not explain how the geometrical parameters and the material properties perform the system. For this purpose in this work we implement all the possible losses performing a parametric analysis of the electromechanical coupling. The proposed approach provides design criteria for efficient energy harvesting AlN systems. We develop the work in the points listed below: in particular we

(i) perform an electromechanical model of piezoelectric AlN cantilever beam including the analysis of modal resonances;

(ii) provide a parametric study of the cantilever losses;

(iii) design an efficient energy harvesting cantilever system by comparing analytical results with numerical ones of a properly designed three dimensional (3D) FEM tool. (iv) we extend the model to an optimized AlN energy harvesting ring layout working at lower frequencies.

$$
\begin{aligned}
& \left.\begin{array}{ccc}
0 & 0 & 0 \\
0 & 0 & 0 \\
0 & 0 & 0 \\
1.25 e^{11} & 0 & 0 \\
0 & 1.25 e^{11} & 0 \\
0 & 0 & 1.25 e^{11}
\end{array}\right)[\mathrm{Pa}] \\
& e=\left(\begin{array}{cccccc}
0 & 0 & 0 & 0 & -0.48 & 0 \\
0 & 0 & 0 & -0.48 & 0 & 0 \\
-0.58 & -0.58 & 1.55 & 0 & 0 & 0
\end{array}\right)\left[\mathrm{C} / \mathrm{m}^{2}\right] \\
& \varepsilon_{r}=\left(\begin{array}{lll}
9 & 0 & 0 \\
0 & 9 & 0 \\
0 & 0 & 9
\end{array}\right)
\end{aligned}
$$

The piezoelectric element is subjected to vertical mechanical vibration $u(t)$ of the seismic mass (proof mass). This

\section{ELECTROMECHANICAL MODELLING AND CANTILEVER MODES}

A complete electromechanical model is illustrated in Figure 1(a) where a transduction system is enclosed in a package. The mechanical deformation of the cantilever device (reported in Fig. 1(b)) leads to an electrical signal using the piezoelectric effect: the external force $F(t)$ shakes the cantilever beam along the vertical direction, the vibration, indicated by the function $u(t)$, will generate through the piezoelectric effect a voltage $\mathrm{V}_{p}$ /current $i$ signal at the output of the system. The system will be matched with an external load $\mathrm{Z}$ in order to transfer the maximum energy. A piezoelectric material (see piezoelectric element of Fig. 1(a)) will convert energy between the mechanical and electrical domains through the following relationships between the stress $\mathbf{T}$, the strain $\mathbf{S}$, the electric $\mathbf{E}$, and the electric displacement field $\mathbf{D}$

$$
\begin{gathered}
\mathbf{T}=c_{E} \mathbf{S}-e^{T} \mathbf{D} \\
\mathbf{D}=e \mathbf{S}-\varepsilon_{r} \mathbf{E}
\end{gathered}
$$

where $c_{E}, e$, and $\varepsilon_{r}$ are the elasticity matrix, the coupling matrix and the relative permittivity matrix, respectively, defined for the AlN material as (FEMLAB libraries)

$$
c_{E}=\left(\begin{array}{ccc}
4.1 e^{11} & 1.49 e^{11} & 9.9 e^{10} \\
1.49 e^{11} & 4.1 e^{11} & 1.49 e^{11} \\
9.9 e^{10} & 1.49 e^{11} & 3.89 e^{11} \\
0 & 0 & 0 \\
0 & 0 & 0 \\
0 & 0 & 0
\end{array}\right.
$$

(a)

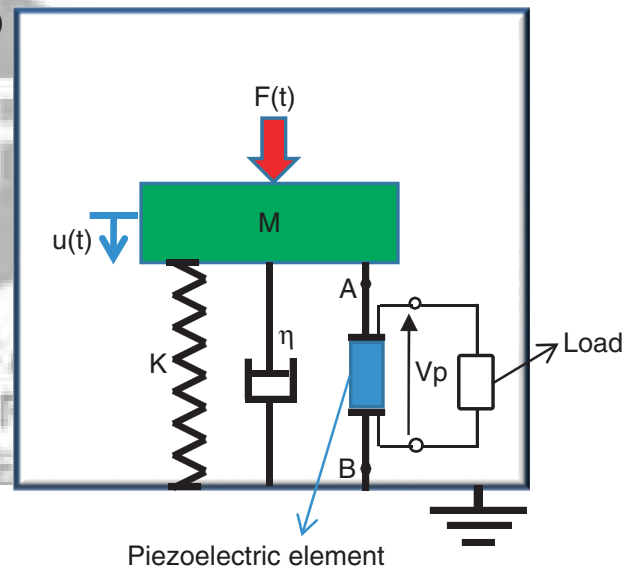

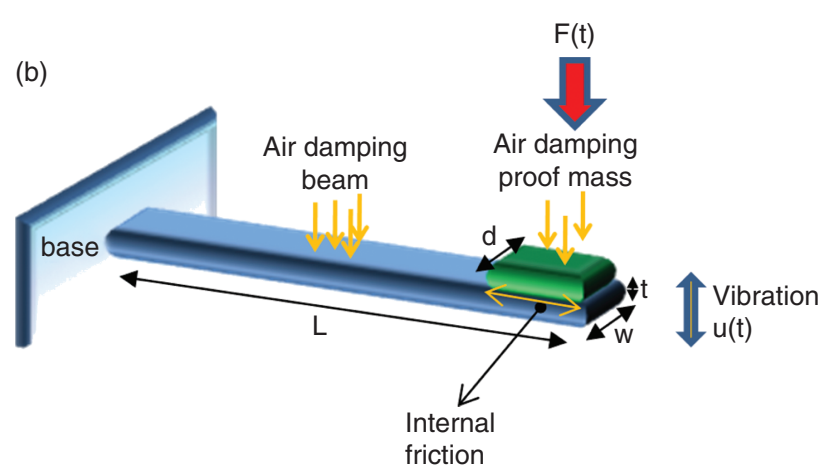

Fig. 1. (a) Equivalent electromechanical model for vibrating energy harvesting cantilever system with proof mass. The losses are modelled by the mechanical resistance indicated by $\eta$. (b) Losses of the piezoelectric cantilever element: damping mechanisms and internal friction of a cantilever in air. 
displacement is solution of the following electromechanical system of equations: ${ }^{14-16}$

$$
\begin{gathered}
i(t)=\alpha \dot{u}(t)-C_{p} \dot{i}(t) \\
K u(t)=F(t)-\alpha \dot{i}(t)-\eta \dot{u}(t)+M \ddot{u}(t)
\end{gathered}
$$

where

- $i(t)$ is the current generated by the vibration flowing in the load of Figure 1(a);

- $\alpha=e_{33} \mathrm{~A} / \mathrm{L}$ is a geometric coefficient linked with piezoelectric coefficient of the material given by Eq. (3), where $\mathrm{A}=\mathrm{w} \cdot \mathrm{L}$ (see Fig. 1(b)) is the cantilever surface;

- $C_{p}=\varepsilon_{33} \mathrm{~A} / \mathrm{t}$ is the free capacitance $(\mathrm{C} / \mathrm{V})$ representing the model of the piezoelectric element of Figure 1(a), where $\varepsilon_{33}$ is given by Eq. (4);

- $K=c_{33} \mathrm{~A} / \mathrm{L}$ is the stiffness calculated from the material stiffness reported in Eq. (2) and device dimensions;

- $M=M_{c}+M_{p}$ is the effective mass of the system where $M_{p}$ is the proof mass (in $\mathrm{g}$ ) and $M_{c}$ is the effective cantilever mass;

- $F(t)=F_{0} \sin (\omega t)$ is the time domain sinusoidal mechanical excitation (see Fig. 1(b)), where $\omega$ is the angular frequency of vibration (in $\mathrm{rad} \mathrm{s}^{-1}$ ), and $F_{0}=M g$ is the constant magnitude ( $g$ is the mass acceleration in $\mathrm{m} / \mathrm{s}^{2}$ );

- $\eta$ is the effective damping factor which includes all the losses reported in Figure 1(b) (internal friction, air damping beam and air damping proof mass).

Note that for a maximum vibrating efficiency $F(t)$ must be in-phase with $u(t)$ and having the same angular frequency of the natural cantilever's resonance. Therefore, in order to fix the working frequency, we calculate the modes (resonance conditions) of the cantilever by means of the following dispersion equation ${ }^{20}$

$$
1+\cos \left(k_{n} L\right) \cosh \left(k_{n} L\right)=0
$$

where $k_{n} L$ are the eigenvalues calculated as illustrated in Figure 2. The calculated eigenvalues related to the first six modes are reported in Table I. Starting from eigenvalues,

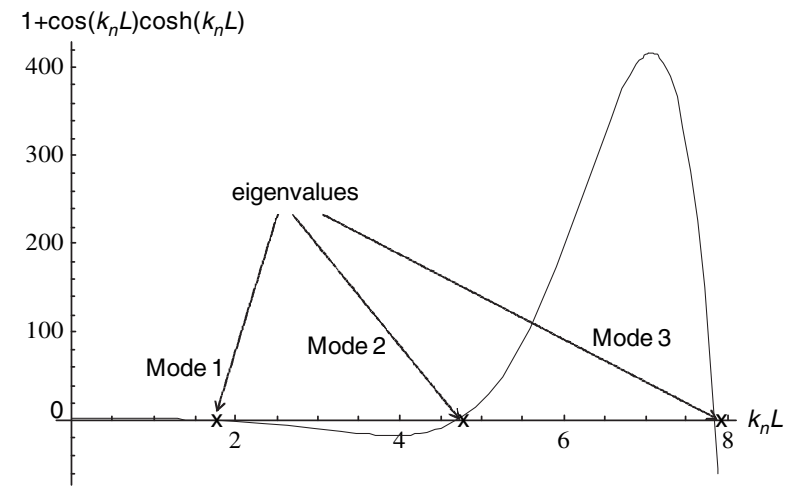

Fig. 2. Dispersion equation versus $k_{n} L$ : the first three eigenvalues related to the first three modes are calculated by the intersection of the function $1+\cos \left(k_{n} L\right) \cosh \left(k_{n} L\right)$ with the axis $1+\cos \left(k_{n} L\right) \cosh \left(k_{n} L\right)=0$.
Table I. Eigenvalues of the first six modes of a uniform cantilever beam.

\begin{tabular}{lc}
\hline Modes & $k_{n} L$ \\
\hline$n=1$ & 1.8751 \\
$n=2$ & 4.694 \\
$n=3$ & 7.855 \\
$n=4$ & 10.996 \\
$n=5$ & 14.1372 \\
$n=6$ & 17.2788 \\
\hline
\end{tabular}

mode shapes for a uniform cantilever beam can be calculated for each mode as

$$
\begin{aligned}
X_{n}(x)= & B_{n}\left[\cosh \left(k_{n} x\right)-\cos \left(k_{n} x\right)\right. \\
& \left.-r_{n}\left(\sinh \left(k_{n} x\right)-\sin \left(k_{n} x\right)\right)\right]
\end{aligned}
$$

where

Ingenta to: $\quad r_{n}=\frac{\cosh \left(k_{n} L\right)+\cos \left(k_{n} L\right)}{\sin \left(k_{n} L\right)+\sin \left(k_{n} L\right)}$

and $B_{n}$ is a modal constant. In Figure 3 are reported the effects of the natural frequency resonances of the first four modes (mode shapes) for a beam with $L=500 \mu \mathrm{m}$. The natural circular frequency of the $n$th mode of a cantilever without proof masses will be given by the following relationship:

$$
k_{n}^{4}=\frac{\rho \omega_{n}^{2}}{E I}
$$

where $E$ is the modulus of elasticity, $I$ is the cross sectional moment of inertia and $\rho$ is the mass per unit length. Moreover, the effective cantilever mass $M_{c}$ will be Ref. [21] calculated by the eigenvalues as

$$
M_{c}=m_{0} \frac{3}{\left(k_{n} L\right)^{4}}
$$

where $m_{0}=\rho A$ ( $\rho$ is the cantilever mass density that for AlN material is equal to $3260 \mathrm{Kg} \mathrm{m}^{-3}$ ).

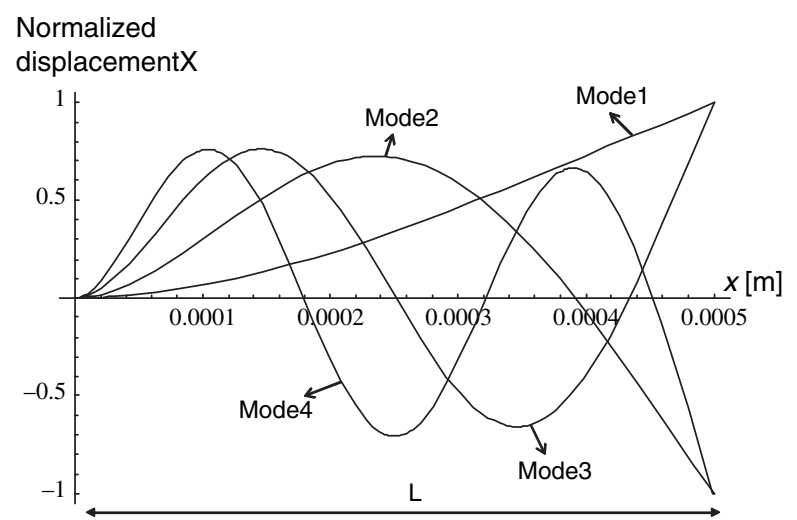

Fig. 3. Normalized beam displacement: mode shapes of the first four modes of vibration. 


\section{IMPLEMENTATION OF CANTILEVER LOSSES AND DESIGN CRITERIA}

The key idea for more power generation is to maximize the energy. The maximum power harvesting, therefore, can be achieved by having a proof mass as heavy as possible unless it result other adverse effects such as losses. Moreover the proof masses are used in order to operate at low frequencies as happens in energy harvesting systems.

The main losses of a cantilever beam with proof mass are reported in Figure 1(b): the damping of the cantilever structure can come from the flapping air, internal friction and beam support losses. The dominant contributors at the MEMS scale are the air damping and internal structure damping. Damping coefficients from the air damping and internal structure damping is determined by the governing equations $^{17}$

$$
\eta=\eta_{\text {air }}+\eta_{\text {internal }}
$$

with

$$
\eta_{\text {air }}=\eta_{\text {air/beam }}+\eta_{\text {air/proofRic }}
$$

being $\eta_{\text {air/beam }}$ the damping effect due to the interaction between air and cantilever, $\eta_{\text {air/proof }}$ the damping effect due to the interaction between air and proof mass, and $\eta_{\text {internal }}$ the internal friction due to interaction between the tip of the cantilever and the proof mass. In particular ${ }^{17}$

$$
\begin{aligned}
\eta_{\text {air } / \text { beam }}= & (1 / w)\left(3 \mu_{a} \pi w+0.75 \pi w^{2}\left(2 \rho_{a} \omega \mu_{a}\right)^{1 / 2}\right) \\
& /\left(2 M \omega_{n}\right)
\end{aligned}
$$

$\eta_{\mathrm{air} / \mathrm{proof}}=\left(3 \mu_{a} \pi d+0.75 \pi d^{2}\left(2 \rho_{a} \omega \mu_{a}\right)^{1 / 2}\right) /\left(2 M \omega_{n}\right)$

$$
\eta_{\text {internal }}=\left(M_{c} /\left(M_{p}+M_{c}\right)\right) * \nu
$$

$\left(\mu_{a}=1.73 \mathrm{~Pa} \cdot \mathrm{s}\right.$ : viscosity of air, $\nu=1.4 \mathrm{~Pa} \cdot \mathrm{s}$ : viscosity of the AlN beam material, $\rho_{a}=1229 \mathrm{Kg} / \mathrm{m}^{3}$ : density of air, $\omega=2 \pi f$ : angular frequency of the external force $F(t)$ ). The goal of the design is to consider the enhancement of the piezoelectric generated power by reducing the losses of the whole system. The design criteria for an efficient system are developed in the points listed below.

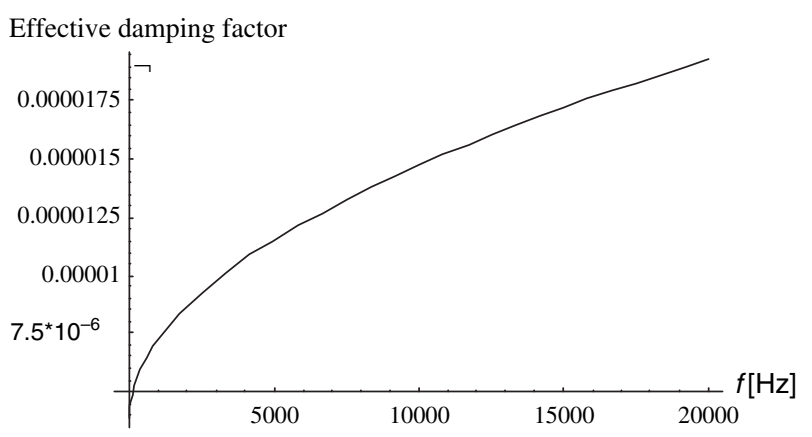

Fig. 4. Total effective damping factor versus the external vibrating frequency for the first mode of a AlN cantilever beam with $w=d=80 \mu \mathrm{m}$, $L=500 \mu \mathrm{m}, t=1 \mu \mathrm{m}$, and $M_{p}=6.5 \mathrm{mg}$.

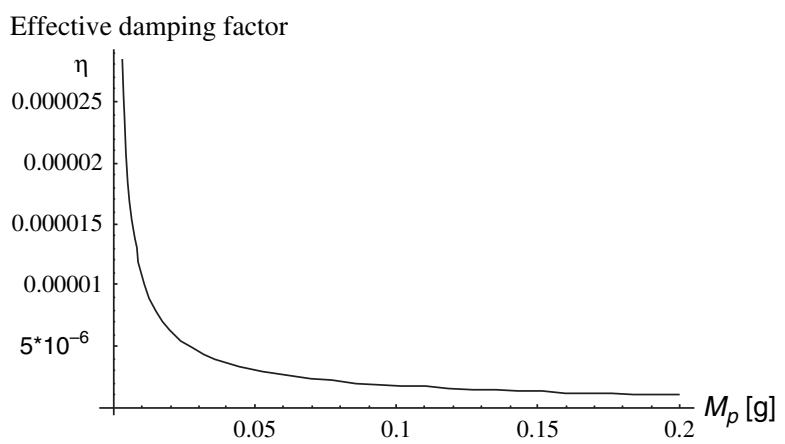

Fig. 5. Total effective damping factor versus the proof Mass $M_{p}$ for the first mode of a AlN cantilever beam with $w=d=80 \mu \mathrm{m}, L=500 \mu \mathrm{m}$, $t=1 \mu \mathrm{m}$, and $\omega=\omega_{1}$.

- Setting of the external vibrating force $(F(t))$. For a maximum cantilever displacement and consecutively for a maximum generated power due to the piezoelectric effect, the external force must have $\omega=\omega_{n}$, where for a system with proof $\operatorname{mass}^{14} \omega_{n}^{2}=K / M$. In Figure 4 is illustrated how the total damping factor increases with respect to the external frequency for an AlN cantilever in the case $\omega \neq \omega_{n} .8: 51$

- Use of a proof mass ${ }_{M p}$. The adding of proof masses allows to operate at lower frequencies if compared with the natural frequencies of the uniform cantilever beam. By Eqs. (13), (14), and (15) we note that the addition of $M_{p}$ (being $M_{p}$ part of total effective mass $M$ ) will decrease the losses due to the internal friction and to the damping losses. In Figure 5 is shown the relationship between the total damping factor and the added proof mass: the total damping factor will decrease with the proof mass, moreover, an high sensitivity of $\eta$ with respect to the parameter $M_{p}$ is observed. According with values reported in literature, proof masses ranging from $105 \mathrm{mg}^{17}$ to $10 \mathrm{~g}^{15}$ can be considered.

- Choice of the geometrical parameters. Observing Eq. (14) the losses decrease with the reduction of the geometrical parameter $d$. As illustrated in Figure 6 (AlN cantilever beam), $\eta$ does not decrease drastically with the parameter $d$ and the same behavior (slow variation) can be observed by varying the parameter $w$ (see relationship

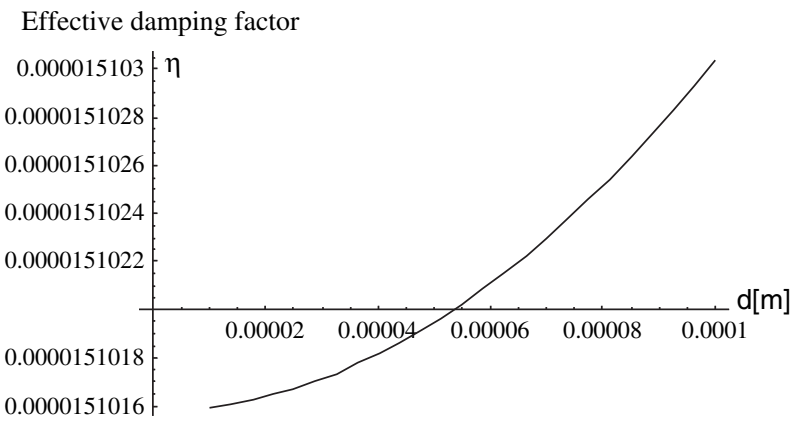

Fig. 6. Total effective damping factor versus proof mass width $d$ for the first mode of a AlN cantilever beam with $w=80 \mu \mathrm{m}, L=500 \mu \mathrm{m}$, $t=1 \mu \mathrm{m}, \omega=\omega_{1}$, and $M_{p}=650 \mathrm{mg}$. 


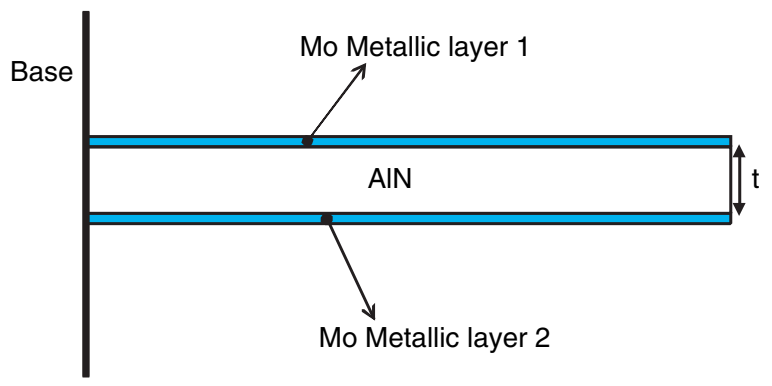

Fig. 7. Cross section of a complete cantilever AlN structure.

of Eq. (13)). In any case the choice of the geometrical parameters must be in agreement with the technological aspects: ${ }^{18,19}$ AlN free standing structure can be fabricated by considering a thickness $t$ ranging from $500 \mathrm{~nm}$ to $1 \mu \mathrm{m}$ for length values $L$ between $200 \mu \mathrm{m}$ and $3 \mathrm{~mm}$. An example of a complete AlN cantilever structure is reported in Figure 7 where two metallic layers (Molybdenum layer) enclose the piezoelectric AlN layer. The layer thickness of the first metallic layer provides the strain properties of

(a)

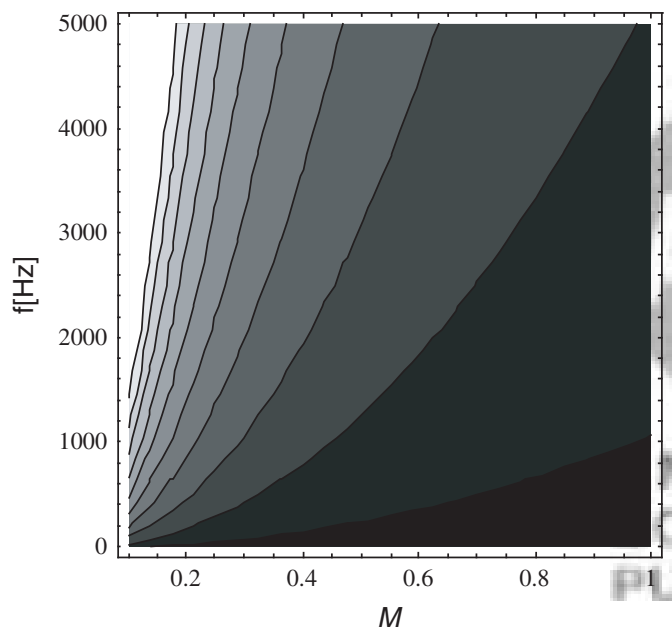

Total damping factor $\eta$

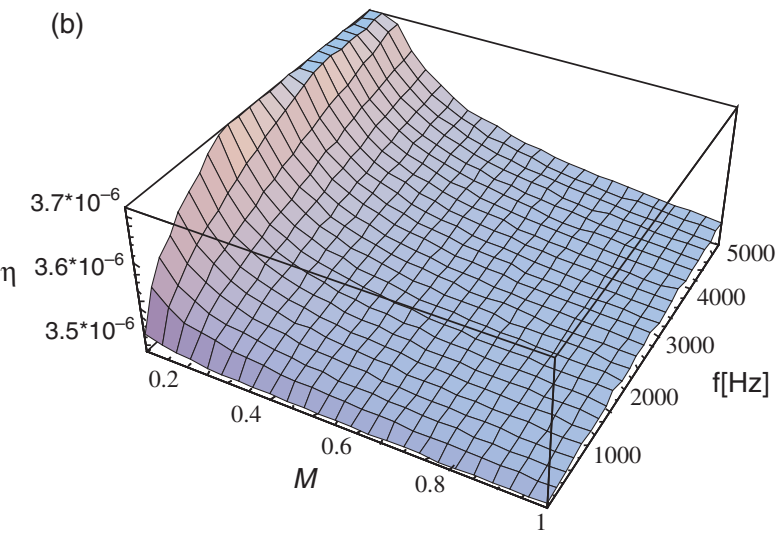

Fig. 8. (a) Contour plot (the white colour corresponds to the higher value of the total damping factor and the black colour corresponds to the lower one) and (b) 3D plot of the total damping factor versus the effective mass $M$ and the vibration force frequency $f$. the whole free standing structure (a typical configuration considers the Mo metallic layer thickness of the order of $\left.50-100 \mathrm{~nm}^{18-19}\right)$.

- Choice of the optimal output load (energy harvesting optimization). In order to transfer a lot of generated power, a proper load must be considered at the output of the system (see Fig. 1(a)). The load must guarantee the higher electromechanical coupling efficiency. The proposed modeling will allow to define the best resistive load.

A complete parametric analysis of the cantilever is illustrated in Figure 8 where is reported the total damping factor versus the frequency of the external force and versus the effective mass $M$ (the effective mass encloses all the most important parameters): a good design can be performed by increasing the effective mass (especially by means of the addition of a proof mass) and by decreasing the working vibrating frequency.

Ingenta to:

\section{ELECTROMECHANICAL MODELLING: RESULT AND DISCUSSIONS}

By implementing the total damping of Eq. (11) in Eq. (5) it is possible to obtain convergent solutions of generated currents $i(t)$ and beam deflections $u(t)$ related to the vibrating system reported in Figures 1(a) and (b). In order to describe the importance of the key parameter $M_{p}$, we apply the model to two designed cantilever structures characterized by the same geometrical dimensions and different proof masses added on the beam tip. In particular, according with technological aspects, ${ }^{18,19}$ and with the parametric analysis, we consider an AlN cantilever with $w=80 \mu \mathrm{m}$, $d=50 \mu \mathrm{m}, L=500 \mu \mathrm{m}$, and $t=1 \mu \mathrm{m}$. Operating with an external angular frequency of $\omega=\omega_{1}$ corresponding to the first resonant mode, we calculate the generated current and the beam deflection in the time domain by considering a proof mass of $6.5 \mathrm{mg}$ (cantilever (1)) and a proof mass of $650 \mathrm{mg}$ (cantilever (2)). Table II reports the differences of the outputs for the two structure by highlighting the importance of the parameter $M_{p}$ for a good design: three order of magnitude of difference are observed for the current $i(t)$ and for the beam deformation $u(t)$, moreover, an order of difference of the effective total damping factor

Table II. Comparison of displacement and currents values between two ALN cantilever structure with the same dimensions and different proof masses. The external force is operating at the resonance frequency of the first mode and the acceleration is $F_{0}=4 \mathrm{~g}$.

\begin{tabular}{lcc}
\hline & Cantilever (1) & Cantilever (2) \\
\hline$M_{p}$ (proof mass) & $6.5 \mathrm{mg}$ & $650 \mathrm{mg}$ \\
$\eta$ (total amping factor) & 0.000367496 & 0.000034467 \\
$f_{1}=2 \pi \omega_{1}$ (working frequency) & $11012.4 \mathrm{~Hz}$ & $1101.22 \mathrm{~Hz}$ \\
$u_{\max }$ (max. beam displacement) & $\sim 4.25 \mathrm{~nm}$ & $\sim 4 \mu \mathrm{m}$ \\
$i_{\max }$ (max. generated current) & $\sim 1.4 \mathrm{pA}$ & $\sim 1.5 \mathrm{nA}$ \\
\hline
\end{tabular}


between the two cantilever is checked. For this purpose we choose as reference structure the cantilever 2 characterized by the higher $M_{p}$ value corresponding to an effective total mass $M=0.65$. The effect of the losses are observed in Figures 9(a) and (b) which illustrate the transient analysis of the output signals: the magnitudes of $u(t)$ and $i(t)$ decrease in the time domain by means of the contribution of $\eta$ which is indicated by the dashed lines. We note that we use for the solution of Figure 9 the initial conditions (of the system (5)) $u(t=0)=0, i(t=0)=0, u^{\prime \prime}(t=0)=0$ which correspond to a no-vibrating cantilever beam at the time $t=0$.

Finally, a good agreement between numerical 3D FEM results and analytical ones is checked in Figure 10 where are reported the normalized generated power $\left(P=V_{p}^{2} / R\right)$ for different accelerations $F_{0}$. The maximum efficiency will be performed when the electromechanical coupling is maximum (energy harvesting optimization). The electromechanical coupling is maximum only for a precise value of the loads $Z=R$. The values of the calculated and simulated loads are reported in Table III. The difference of few Ohms between the 3D FEM numerical and the analytical model indicates the accuracy of the proposed modeling. The cantilever structure is simulated by a properly designed FEM tool which discretizes the cantilever by tetrahedrons and implements Eqs. (1)-(4). We note that, as shown by the trend of Figure 10, it is possible to enhance the generated currents by increasing again the input acceleration or by designing array of coupled cantilever characterized by the same dimensions and operating at the same frequency.

\section{ANALYTICAL MODELING OF RING LAYOUTS WORKING AT LOW FREQUENCIES}

According with the actual technology, ${ }^{19}$ it is possible to control the stress/strain properties (and consecutively the radius of curvature) of an AlN cantilever by means of the Mo layer 1 thickness (the thickness is controlled by the RF
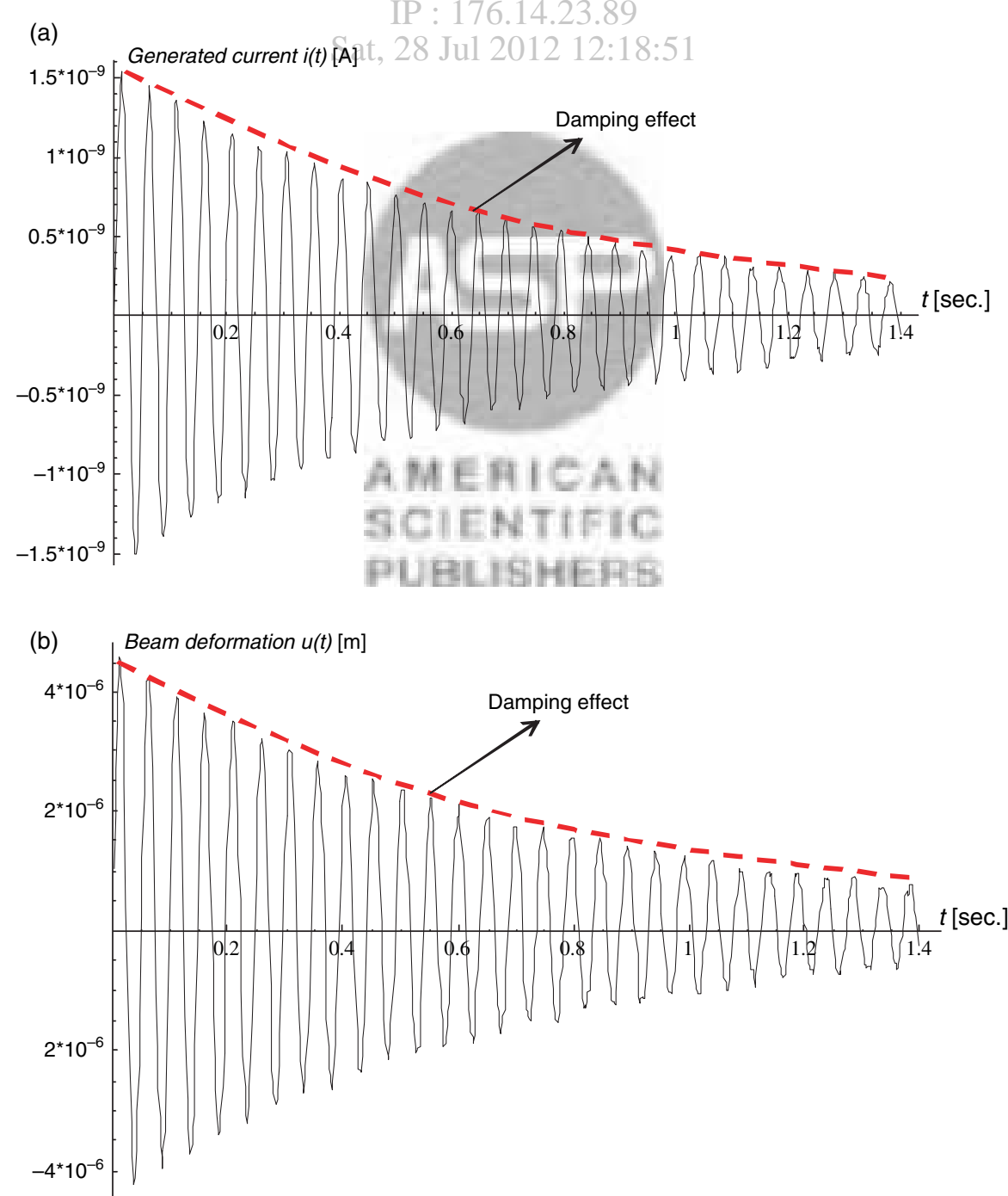

Fig. 9. Cantilever (2): (a) time domain current generated by the external force, and, (b) time domain beam deformation. 


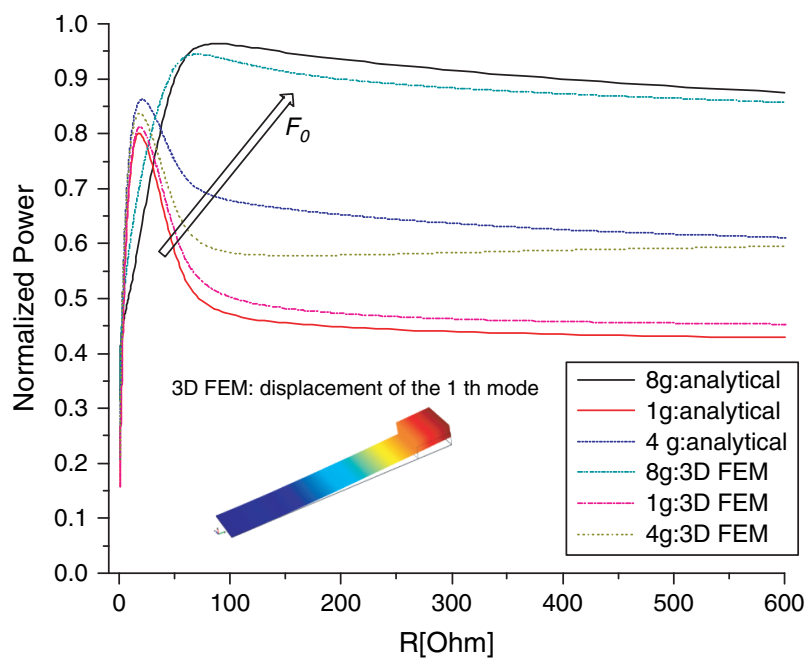

Fig. 10. Comparison between numerical (3D FEM) and analytical results: normalized generated powers versus the load $(Z=R)$ for different accelerations $F_{0}$. Inset: 3D FEM resonance beam shape of the 1 th mode.

sputtering machine). The control of the stress/strain properties allow to obtain Mo/AlN/Mo ring layouts. In order to study the piezoelectric behavior of the ring layouts, we implement the following equation system containing the strain-charge equation form and the charge equation of electrostatics

$$
\begin{gathered}
\left\{\begin{array}{c}
S_{r r} \\
S_{\theta \theta} \\
S_{z z} \\
S_{\theta z} \\
S_{z r} \\
S_{r \theta}
\end{array}\right\}=s_{E}\left\{\begin{array}{l}
T_{r r} \\
T_{\theta \theta} \\
T_{\theta z} \\
T_{z r} \\
T_{r \theta}
\end{array}\right\}+d\left\{\begin{array}{l}
E_{r} \\
E_{\theta} \\
E_{z}
\end{array}\right\} \\
\left\{\begin{array}{l}
D_{r} \\
D_{\theta} \\
D_{z}
\end{array}\right\}=d^{T}\left\{\begin{array}{l}
T_{r r} \\
T_{\theta \theta} \\
T_{z z} \\
T_{\theta z} \\
T_{z r} \\
T_{r \theta}
\end{array}\right\}-\varepsilon_{r}^{T}\left\{\begin{array}{l}
E_{r} \\
E_{\theta} \\
E_{z}
\end{array}\right\} \\
\frac{\partial D_{r}}{\partial r}+\frac{1}{r} \frac{\partial D_{\theta}}{\partial \theta}+\frac{1}{r} D_{r}+\frac{\partial D_{z}}{\partial z}=0
\end{gathered}
$$

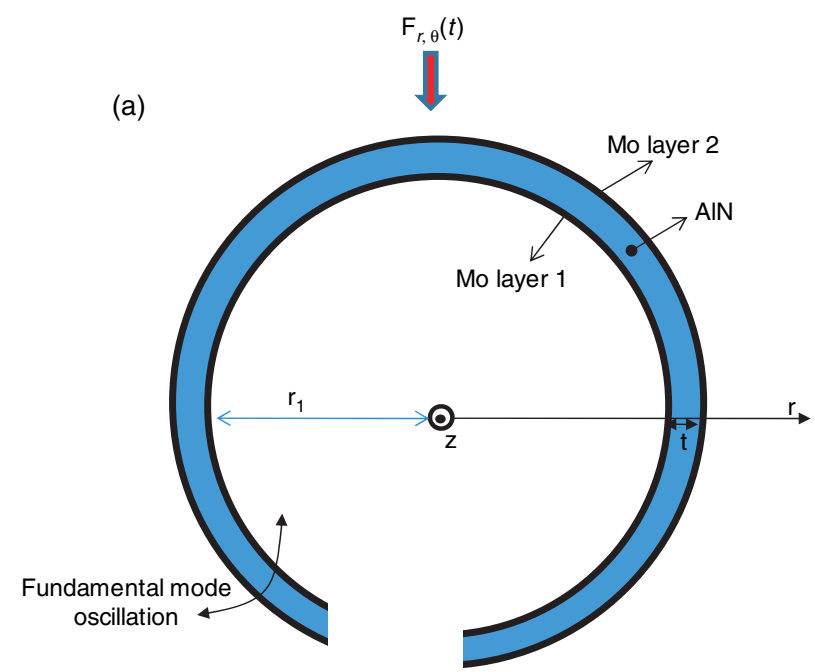

(b)

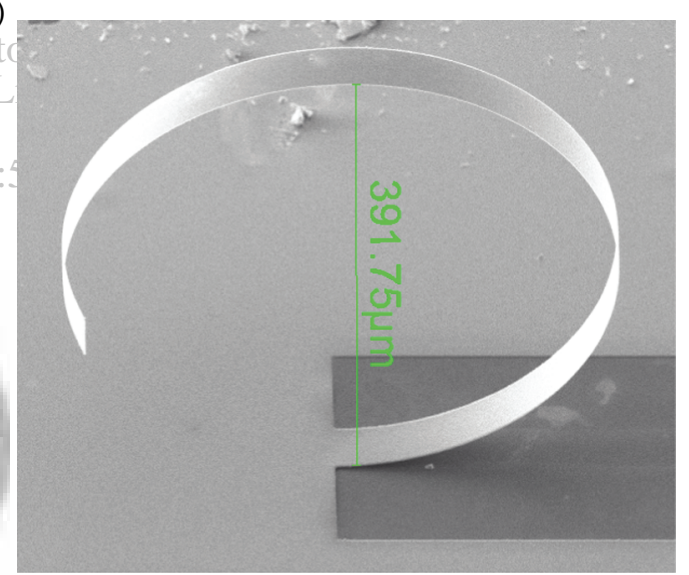

Fig. 11. (a) Piezoelectric ring layout. (b) Scanning electron microscopes (SEM) image of a fabricated Mo/AlN/Mo ring.

with the compliance matrix $\boldsymbol{s}_{E}$ and the coupling matrix $\boldsymbol{d}^{T}$ with the compliance matrix $s_{E}$ and
PUBLISI are given (AIN FEMLAB libraries)

$$
s_{E}=\left(\begin{array}{ccc}
2.85842 e^{-12} & -8.99652 e^{-13} & -3.82867 e^{-13} \\
-8.99652 e^{-13} & 3.11659 e^{-12} & -9.64799 e^{-13} \\
-3.82867 e^{-13} & -9.64799 e^{-12} & 3.03768 e^{-12} \\
0 & 0 & 0 \\
0 & 0 & 0 \\
0 & 0 & 0
\end{array}\right.
$$

$$
\left.\begin{array}{ccc}
0 & 0 & 0 \\
0 & 0 & 0 \\
0 & 0 & 0 \\
8 e^{-12} & 0 & 0 \\
0 & 8 e^{-12} & 0 \\
0 & 0 & 8 e^{-12}
\end{array}\right)[1 / \mathrm{Pa}]
$$

Table III. Loads for efficient electromechanical coupling.

\begin{tabular}{lcc}
\hline$F_{0}$ & 3D FEM R $[\Omega]$ & Analytical R $[\Omega]$ \\
\hline $1 \mathrm{~g}$ & 29 & 26 \\
$4 \mathrm{~g}$ & 39 & 36 \\
$8 \mathrm{~g}$ & 71 & 69 \\
\hline
\end{tabular}




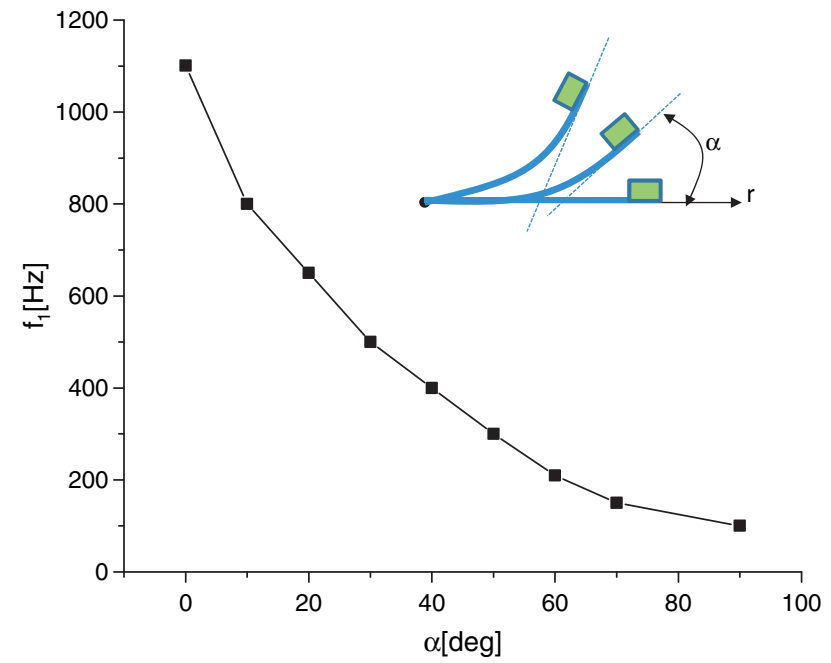

Fig. 12. Cantilever beam 2 of Table II: piezoelectric resonant frequency $f_{1}$ of the fundamental mode versus the $\alpha$-angle. Inset: the radius of curvature is expressed in terms of the angle $\alpha$.

$$
d^{T}=\left(\begin{array}{ccc}
0 & 0 & 0 \\
0 & 0 & 0 \\
-1.72953 e^{-12} & -2.78126 e^{-12} & 5.49006 e^{-1} \\
0 & -3.84 e^{-12} & 0 \\
-3.84 e^{-12} & 0 & 0 \\
0 & 0 & 0
\end{array}\right)[\mathrm{C} / \mathrm{N}]
$$

respectively.

As illustrated in Figure 11, an applied force $F(r, \theta, t)$ will generate a displacement $u_{r}(r, \theta, t)$ and $u_{\theta}(r, \theta, t)$. Due to the lower resonant frequency of the fundamental mode $f_{1}$, we simulate the cantilever beam 2 of Table II ( $L=500 \mu \mathrm{m}, w=80 \mu \mathrm{m}, t=50 \mu \mathrm{m}, d=50 \mu \mathrm{m})$ by changing the radius of curvature. The analytical solutions of the set of Eqs. (16) and (5) provides in Figure 12 the piezoelectric fundamental resonant frequency $f_{1}$ versus the $\alpha$ angle defined by the $r$-axis and the tangent to the tip (see the inset of Fig. 12).

These results highlight the importance of the radius of curvature about the possibility to work at lower vibration frequency as required in many energy harvesting cases.

\section{CONCLUSION}

We present in this work an accurate modelling of a vibrating energy harvesting system based on AlN cantilevers. The modeling includes all the possible losses and highlights the importance of the proof mass during the design approach. A good agreement between 3D FEM results and analytical ones confirms the accuracy of the proposed model. The model explains in details how the geometrical parameters and the material properties perform the response of the system. The model is extended to a ring layout by obtaining lower resonant frequency of the fundamental mode. A study of complex arrays of rings/cantilever systems and experimental aspects are under investigation.

\section{References}

1. S. Roundy, E. S. Leland, J. Baker, E. Carleton, E. Reilly, E. Lai, B. Otis, J. M. Rabaey, P. K. Wright, and V. Sundararajan, IEEE Pervasive Computing 4, 28 (2005).

2. H. A. Sodano, Shock and Vibration Digest 36, 197 (2004).

3. B. S. Lee, S. C. Lin, W. J. Wu, X. Y. Wang, P. Z. Chang, and C. K. Lee, Journal of Micromechanics and Microengineering 19, 065014 (2009).

4. W. J. Choi, Y. Jeon, J.-H. Jeong, R. Sood, and S. G. Kim, Journal of Electroceramics 17, 543 (2006).

5. B. S. Lee, W. J. Wu, W. P. Shih, D. Vasic, and F. Costa, IEEE Ultrasonic Symosium 1, 1598 (2007).

6. S. Roundy and P. K. Wright, Smart Materials and Structures 13, 1131 (2004).

7. Y. B. Jeon, R. Sood, J. H. Jeong, and S. G. Kim, Sensors Actuators A 122, 16 (2005).

8. C. B. Williams and R. B. Yates, Sensors Actuators A 52, 8 (1996).

9. D. Shen, J-H. Park, J. Ajitsaria, S.-Y. Choe, H. C. Wikle, and D.-J. Kim, Journal of Micromechanics and Microengineering 18, 055017 (2008).

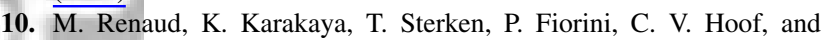
R. Puers, Sensors Actuators A 145, 380 (2008).

11. S. Roundy, P. K. Wright, and J. Rabaey, Comuter Communications 26, 1131 (2003).

12. M. Marzencki, Y. Ammar, and S. Basrour, Sensors Actuators A 145, 363 (2008).

13. R. J. M. Vullers, R. Van Schaijk, I. Doms, C. Van Hoof, and R. Mertens, Solid State Electronics 53, 684 (2009).

14. Y. C. Shu and I. C. Lien, Smart Matererials and Structures 15, 1499 (2006).

15. H. Boussetta, M. Marzencki, Y. Ammar, and A. Basrour, IEEE Behavioral Modeling and Simulation Workshop 978, 126 (2007).

16. S. R. Platt, S. Farritor, and H. Haider, IEEE Transaction on Mechatronics 10, 455 (2005).

17. W. C. Choi, Y. H. Jeong, R. Sood, and S. G. Kim, Journal of Electroceramics 17, 543 (2006).

18. C. Giordano, I. Ingrosso, M. T. Todaro, G. Maruccio, S. De Guido, R. Cingolani, A. Passaseo, and M. De Vittorio, Journal of Microelectronic Engineering 86, 1204 (2009).

19. A. Massaro, I. Ingrosso, C. Giordano, M. T. Todaro, R. Cingolani, M. De Vittorio, and A. Passaseo, IEEE EuMC Proceeding 1, 1646 (2009).

20. D. W. Dareing, T. Thundat, S. Jeon, and M. Nicholson, Journal of Applied Physics 97, 084902 (2005).

21. J. Teva, G. Abadal, Z. J. Davis, J. Verd, X. Borrisè, A. Boisen, F. P. Murano, and N. Barniol, Ultramicroscopy 100, 800 (2004).

Received: 11 January 2011. Accepted: 31 January 2011. 